\title{
Per Oral Endoscopic Myotomy in Children with Achalasia Cardia
}

\author{
Zaheer Nabi, ${ }^{1}$ Mohan Ramchandani, ${ }^{1}$ D Nageshwar Reddy, ${ }^{1 *}$ Santosh Darisetty, ${ }^{2}$ Rama Kotla, ${ }^{2}$ Rakesh Kalapala, ${ }^{1}$ and \\ Radhika Chavan ${ }^{1}$
}

Departments of ${ }^{1}$ Gastroenterology and ${ }^{2}$ Anesthesiology, Asian Institute of Gastroenterology, Hyderabad, India

\section{Background/Aims}

Achalasia cardia (AC) is a motility disorder, characterized by impaired lower esophageal sphincter relaxation and absence of esophageal peristalsis. AC is rare in children with unclear optimum management strategies. Per oral endoscopic myotomy (POEM) is a novel technique for management of achalasia with encouraging results in adult patients. The efficacy and safety of POEM is not known for pediatric AC. The aim of our study was to evaluate the safety and efficacy of POEM in children with achalasia cardia

\section{Methods}

The data of all children ( $<18$ years) who underwent POEM at our center was retrospectively analysed. Symptoms were analysed using a validated score (Eckardt score) at regular predefined intervals. Objective parameters including high-resolution manometry, timed barium swallow and esophagogastroduodenoscopy were assessed before the procedure and at 1-year follow-up. Clinical success was defined as an Eckardt score $\leq 3$.

\section{Results}

A total of 15 children underwent POEM during the specified period. Ten out of 15 (10/15) completed 1-year follow-up. Median operative time was 100 (38-240) minutes. Mean pre and post procedure LES pressure were $36.64 \pm 11.08 \mathrm{mmHg}$ and $15.65 \pm 5.73$ $\mathrm{mmHg}$, respectively $(P=0.001)$. Mean Eckardt score before and after the POEM was $7.32 \pm 1.42$ and $1.74 \pm 0.67$, respectively $(P=$ 0.001). Mean percentage improvement in barium emptying at 5 minutes was $63.70 \pm 4.46 \%$. All children had complete resolution of symptoms at 1 year. Median weight gain of children at 1 year was $0.65 \mathrm{~kg}$ (range, 0.0-4.6).

\section{Conclusions}

POEM is safe and effective for children and adolescents with achalasia. Future trials with larger sample size are warranted to establish its efficacy in pediatric AC.

(J Neurogastroenterol Motil 2016;22:613-619)

\section{Key Words}

Achalasia cardia; Child; Endoscopic surgical procedure

Received: October 27, 2015 Revised: February 23, 2016 Accepted: March 5, 2016

(a) This is an Open Access article distributed under the terms of the Creative Commons Attribution Non-Commercial License (http://creativecommons. org/licenses/by-nc/4.0) which permits unrestricted non-commercial use, distribution, and reproduction in any medium, provided the original work is properly cited.

${ }^{*}$ Correspondence: D Nageshwar Reddy, MD

Department of Gastroenterology, Asian Institute of Gastroenterology, 6-3-661, Somajiguda Hyderabad 500082, India Tel: +91-40-23378888, Fax: +91-40-23324255, E-mail: aigindia@yahoo.co.in 


\section{Introduction}

Achalasia cardia (AC) is a rare neurodegenerative disease characterized by the degeneration of inhibitory myenteric plexus of the lower esophageal sphincter (LES) and esophageal body. The imbalance of inhibitory and excitatory neurons results in failure of LES relaxation and absence of peristalsis during swallowing. ${ }^{1}$ Achalasia is very rare in children. ${ }^{2}$ Unfortunately, very few epidemiological studies have been conducted for achalasia in pediatric patients. ${ }^{4}$ In fact, there is no population based epidemiological study of achalasia in children or adolescents in India. Previous studies show an incidence of less than $0.1 / 10^{5}$ population/year. ${ }^{3}$ However the incidence of pediatric achalasia appears to have increased over the last decade (about $0.18 / 10^{5}$ population/year). ${ }^{3,4}$

Older children usually present with typical symptoms like dysphagia and vomiting, whereas younger children and infants often present with atypical symptoms like recurrent pneumonia, feeding difficulties, and cough. ${ }^{5}$

The treatment options for achalasia cardia in the pediatric population include medical (calcium channel blockers, nitrates), endoscopic (botulinum toxin injection and pneumatic balloon dilatation $[\mathrm{PBD}]$ ), and surgical (laparoscopic Heller's cardiomyotomy) treatments. However, except for PBD and laparoscopic Heller's myotomy (LHM), experience and efficacy of other therapies is limited in children. Therefore, there is an unmet need for an optimum endoscopic treatment modality for achalasia in the pediatric population.

Recently, per oral endoscopic myotomy (POEM) has gained widespread acceptance for the treatment of adult patients with achalasia. Short term results demonstrate good safety and efficacy of this novel technique. ${ }^{6}$ Though the experience in pediatric patients is limited, it is a potential alternative to LHM, as it is less invasive with faster recovery and good safety.

In this series, we describe 15 children with esophageal achalasia who underwent POEM at our center.

\section{Materials and Methods}

We retrospectively analysed the data of all the children undergoing POEM at our center. The study protocol was reviewed and approved by our review board (AIG/AHF IRB: 34/2015). Each of the endoscopist performing POEM in children had prior experience of at least 20 adult cases. Achalasia cardia was diagnosed with standard diagnostic modalities including timed barium esophagogram $(\mathrm{TBE})^{7}$, high-resolution manometry (HRM) and esophagogastroduodenoscopy (EGD) in all the children. Children with contraindications to undergo general anaesthesia or whose parents did not give consent for the procedure were excluded from this study. A history of previous endoscopic (including repeated balloon dilatation and botulinum toxin injection) or surgical treatment (open or LHM) were not considered as exclusion criteria. Children with esophageal candidiasis on EGD were treated with an oral antifungal for at least 1 week prior to the procedure. Pre-operative symptom evaluation was done using Eckardt score. ${ }^{8}$ HRM was performed with a 16 channel water perfused catheter (Dent sleeve International Limited, manufactured by Mui Scientific, Ontario, Canada). The data were analyzed using Trace $1.2 \mathrm{~V}$ software (Geoff Hebbard, Royal Melbourne Hospital, Victoria, Australia). The subtypes of the AC were defined according to the Chicago classification. ${ }^{9}$ Any complication requiring additional procedures or prolonged hospital stay ( $>3$ days) was considered as morbidity. All children were followed up at 3 months, 6 months, and 1 year after the procedure. At the 3 and 6 months follow-up visit, physical examination and symptom evaluation (Eckardt score) was done. Esophageal manometry, EGD and TBE were done at 1-year follow-up. A postoperative Eckardt score $\leq 3$ was considered as a successful outcome.

\section{Technique}

All children, posted for the POEM procedure were instructed to take liquid diet for 1 day prior to the procedure. Prophylactic antibiotics were given on the day of the procedure and continued during the entire period of hospitalization. The procedure was carried out under general anaesthesia in the supine position. Standard EGD endoscope (Olympus GIF HQ 190; Olympus Corp, Tokyo, Japan) was used for the procedure. A tapered tip transparent cap (DH-28GR; Fujifilm, Tokyo, Japan) was fitted onto the distal end of the scope to facilitate submucosal dissection. Children with a prior history of myotomy (LHM) underwent posterior myotomy (at 5 o'clock position). The choice of site of myotomy (anterior, at 2 o'clock position vs posterior, at 5 o'clock position) ${ }^{10}$ was left to the endoscopist's preference in the rest of the children. A 23 gauge sclerotherapy needle was used to create a submucosal wheal by injecting normal saline with indigo carmine submucosally at least 3-4 $\mathrm{cm}$ proximal to the high-pressure zone identified at the pre-operative HRM (Fig. 1A). ${ }^{11}$ Subsequently a small (3-4 mm) mucosal incision was made using a needle knife which was further enlarged up to $2 \mathrm{~cm}$ in length by an insulated tip knife (KD-611L; Olympus Corp) (Fig. 1B). In the next step, a submucosal tunnel was created 
A

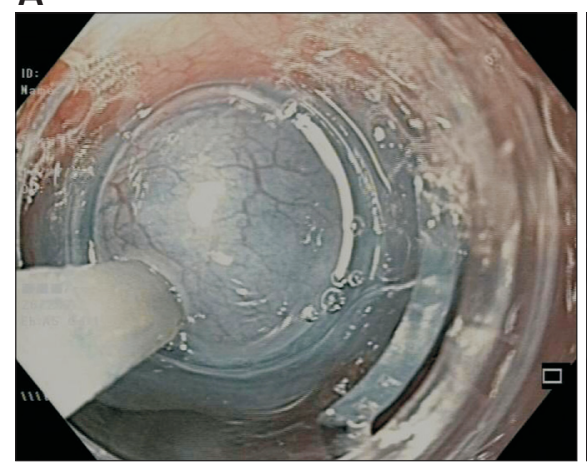

C

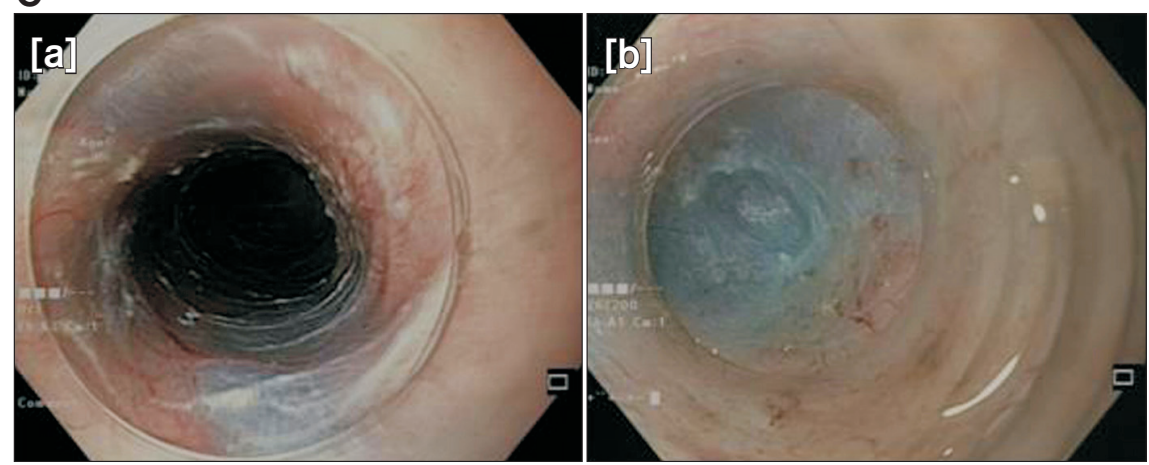

B

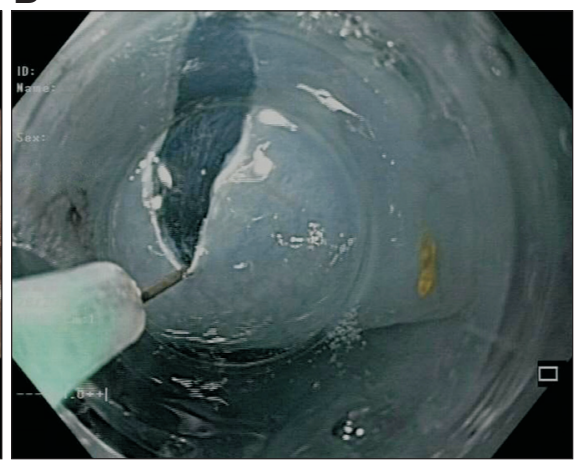

D

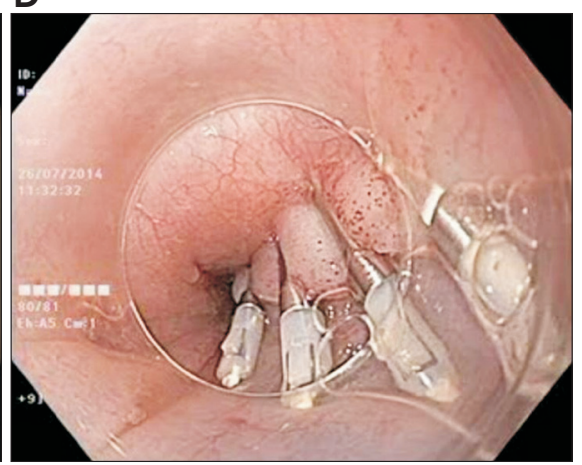

Figure 1. The sequential steps of per oral endoscopic myotomy. (A) Lifting of the mucosa away from the submucosa by submucosal injection using a sclerotherapy needle. (B) Initial small mucosal incision made with needle knife followed by enlarging the incision with insulated tip knife. (C) [a] Submucosal tunnel on the esophageal side (mucosa is along 5 to 11 o'clock and muscle along 11 to 5 o'clock) and [b] submucosal tunnel along the gastric end of tunnel. (D) Closure of mucosal incision with endoclips after completion of myotomy.

using spray coagulation (Fig. 1C). Carbon dioxide $\left(\mathrm{CO}_{2}\right)$ insufflation was used throughout the procedure (UCR; Olympus Corp) (Fig. 1C). All the cases were done using a low flow gas tube (MAJ 1742; Olympus Corp) attached to a $\mathrm{CO}_{2}$ insufflator (UCR; Olympus Corp). A triangular tip knife (KD-640L; Olympus Corp) with a spray coagulation mode was used for the submucosal dissection and myotomy. The settings on the electrosurgical unit (VIO300D; ERBE, Tübingen, Germany) during dissection and myotomy were identical ie, ENDO CUT Q at $50 \mathrm{~W}$, effect 3. The tunnel was extended to least $2 \mathrm{~cm}$ onto the gastric cardia past the gastroesophageal junction (GEJ). Intervening blood vessels or bleeding during dissection was managed by coagulation forceps (Coagrasper G, FD-412LR, Olympus, Japan) using the soft coagulation mode (80W, effect 5). The gastric extent of the submucosal tunnel was confirmed by visualizing the blanched gastric mucosa. The myotomy was done in a proximal-to-distal fashion. Circular muscle only myotomy was performed in the upper esophagus, taking care to preserve the longitudinal muscle layers. Full thickness myotomy was performed at the lower end of the esophagus and cardia. Adequacy of the myotomy was established by smooth passage of the endoscope through the GEJ and a retroflexed evaluation of the LES. The mucosal incision was then closed from the distal to proximal end using standard endoscopic clips (EZ Clip, HX-610090L; Olympus Corp) (Fig. 1D). All children were evaluated with a water soluble contrast esophagogram on the second postoperative day (Fig. 2A and 2B). If normal, they were then started on a pureed diet and subsequently discharged. The pureed diet was continued for 1 week. Subsequently a regular diet was allowed.

\section{Statistical Methods}

The data was prospectively collected and comparison of pre and post procedure parameters was done. Data are presented as mean \pm standard deviation. Student's pair $t$ test was used for continuous variables and proportion test for categorical variable. A $P$-value of $<0.05$ was considered as statistically significant. 

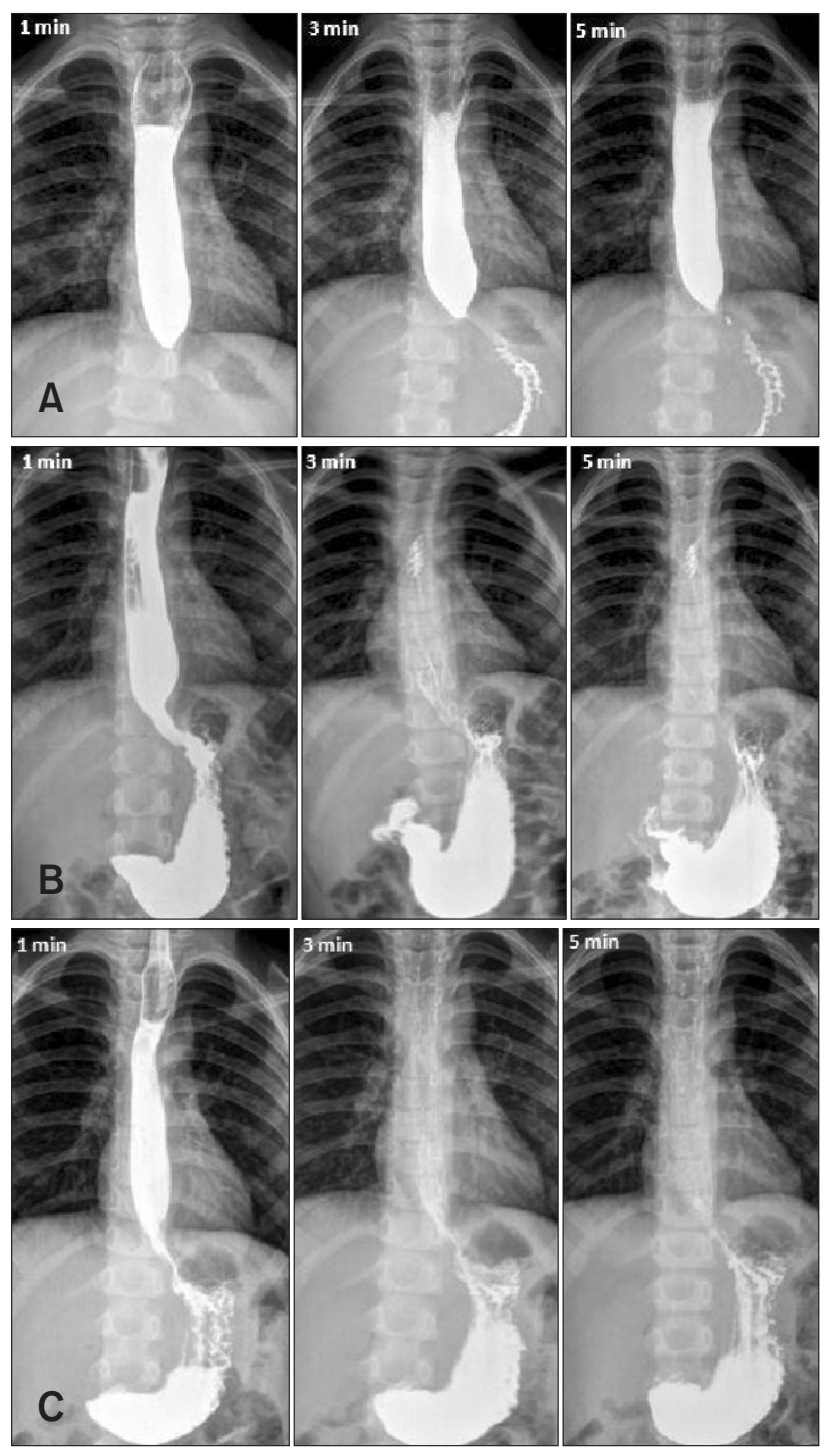

Figure 2. Assessment of the esophageal emptying with timed barium esophagogram. (A) Timed barium esophagogram before per oral endoscopic myotomy (POEM). Note the significant stasis of barium column at 1, 3, and 5 minutes. (B) Timed barium esophagogram after POEM on day 2. Note the free flow of barium across the gastroesophageal junction at 1 minute and nearly complete emptying of barium column at 3 and 5 minutes. (C) Timed barium esophagogram of the same child at about one year after POEM. Excellent barium emptying is seen at 3 and 5 minutes.

\section{Results}

A total of 15 children (median age 14 years, range 9 to 18 years) with achalasia cardia underwent POEM in our department during the study period (October 2013 to April 2015) (Table 1).
Table 1. Characteristics of Children Undergoing Per Oral Endoscopic Myotomy

\begin{tabular}{lc}
\hline Number of patients & 15 \\
Achalasia cardia (n) & \\
Type I & 10 \\
Type II & 1 \\
Type III & $14(9-18)$ \\
Age (median [range], yr) $(\mathrm{n}=15)$ & $9: 6$ \\
Male:female & $29(5-96)$ \\
Disease duration (median [range], mo) & \\
Previous therapy & $0(0.0 \%)$ \\
Botulinum toxin injection & $6(40.0 \%)$ \\
Pneumatic balloon dilatation & $1(6.7 \%)$ \\
Heller's myotomy & $1(0.0 \%)$ \\
Pneumatic dilatation and Heller's, both &
\end{tabular}

Table 2. Technical Details of the Procedure and Adverse Events

\begin{tabular}{lc}
\hline Site of Myotomy & 13 \\
Anterior & 2 \\
Posterior & $100(38-240)$ \\
Operating time (median [range], min) & $12(6-16)$ \\
Length of myotomy (median [range], cm) & $8.0(4-12)$ \\
Esophageal (median [range], cm) & $2(2-3)$ \\
Gastric (median [range], cm) & $15 / 15(100 \%)$ \\
Technical success & \\
Adverse events & $0(0.0 \%)$ \\
Clinically significant bleeding & $1(6.7 \%)$ \\
Mucosal injury & $2(13.3 \%)$ \\
Self limiting subcutaneous emphysema & $1(6.7 \%)$ \\
Capnoperitoneum requiring drainage & $0(0.0 \%)$ \\
Capnothorax & $3(20.0 \%)$ \\
Retroperitoneal air requiring temporary & \\
$\quad$ stoppage the procedure & $2.0(1.0-4.0)$ \\
Feeding (median [range], day) & $2.7(2.0-5.0)$ \\
Hospital stay (mean [range], day) & 0 \\
30 day readmission rate & $5(5-8)$ \\
Number of clips (median [range]) & 0 \\
Perioperative complications 0-30 days & \\
\hline
\end{tabular}

The types of achalasia cardia as per the Chicago classification were type I $(\mathrm{n}=4)$, type II $(\mathrm{n}=10)$, and type III $(\mathrm{n}=1)$. Six patients $(40.0 \%)$ were previously treated by PBD and one patient had a history of LHM. One patient had undergone both LHM and PBD. Median pre-procedure Eckardt score was 8 (range, 5-9). Median LES pressure prior to the procedure was $35.2 \mathrm{mmHg}$ (range, 22$55 \mathrm{mmHg}$ ). POEM was successfully completed in all the 15 children $(100.0 \%)$ patients with AC (Table 2). The myotomy was performed via an anterior approach (2 o'clock) in 13 children (86.6\%) 
and posterior approach (5 o'clock position) in 2 children. Median procedure time was 100 minutes (range, 38-240 minutes). Median length of total myotomy was $12 \mathrm{~cm}$ (range, 6-16 cm). The median length of myotomy on the esophageal side and gastric side was 8.0 $\mathrm{cm}$ (range, $5-12 \mathrm{~cm}$ ) and $2.0 \mathrm{~cm}$ (range, 2-3), respectively. Intraoperative gas related events included transient subcutaneous emphysema in 2 children (13.3\%), retroperitoneal air in 3 children (20.0\%), and capnoperitoneum in 1 child $(6.7 \%)$. The child with capnoperitoneum was managed by draining the $\mathrm{CO}_{2}$ using conventional intravenous canula, whereas the procedure was temporarily stopped for few minutes in those with retroperitoneal $\mathrm{CO}_{2}$. POEM was resumed in these children after fluoroscopic confirmation of resorption of retroperitoneal $\mathrm{CO}_{2}$. Inadvertent mucosal injury was seen in 1 child $(6.7 \%)$ which was successfully closed using hemoclips at the end of the procedure. Thirteen children completed 6 months and 10 children completed 1-year follow-up (median, 15 months; range, 12-20 months). At 6 months follow-up, all 13 patients had an Eckardt score < 3 with clinical success of $100 \%$. Mean Eckardt score decreased significantly from $6.26 \pm 1.43$ at baseline to 1.26 $\pm 0.79(P=0.001)$ at 6 months. No adverse events were recorded during follow-up.

In addition to symptom analysis, objective parameters including HRM, EGD, and TBE were also assessed at 1 year of follow-up (Table 3). Ten out of 15 children completed 1-year follow-up and the clinical success was $100 \%$ (10/10). Mean Eckardt score was 7.32 \pm 1.42 prior to $\mathrm{POEM}$ and $1.74 \pm 0.67$ after the $\operatorname{POEM}(P=$ 0.001). HRM was available for 10 children who completed 1-year follow-up. Pre-procedure mean LES pressure was $36.64 \pm 11.08$ $\mathrm{mmHg}$ and post procedure mean LES pressure was $15.65 \pm 5.70$ $\mathrm{mmHg}(P=0.001)$. Mean integrated relaxation pressure before and after the procedure was $24.96 \pm 5.42 \mathrm{mmHg}$ and $9.48 \pm 1.95$ mmHg respectively $(P=0.001)$.

Mean height of barium column (TBE) at 5 minutes was 12.48 $\pm 1.48 \mathrm{~cm}$ preoperatively and $4.60 \pm 0.77 \mathrm{~cm}$ postoperatively $(P$ $=0.001)$. Mean percentage improvement in barium emptying at 5 minutes was $63.70 \pm 4.46 \%$.
At 1-year follow-up 3 patients (30.0\%) had gastroesophageal reflux as assessed by clinical history and symptom analysis. EGD revealed erosive esophagitis in 2/10 (20.0\%) patients (Los Angeles grade $\mathrm{A}$ in 1 patient and grade $\mathrm{B}$ in 1 patient). None of the patients had grade $\mathrm{C}$ or $\mathrm{D}$ esophagitis. The median weight gain in children who completed 1-year follow-up was $0.65 \mathrm{~kg}$ (range, 0.0-4.6).

\section{Discussion}

In the present series, we found POEM to be safe and effective for the treatment of AC in children. The response to POEM appears durable with lack of significant adverse effects.

Achalasia is rare in children, accounting for the lack of large randomized controlled studies on its management. The management of achalasia in children is still unclear and there is no established treatment protocol in this population. ${ }^{12}$

Currently the management options include endoscopic and surgical modalities. The endoscopic options are PBD and intraesophageal botulinum toxin injection. PBD has a reported success between $70-90 \%$ in published case series. ${ }^{13,14}$ Many experts prefer PBD as initial therapy for older children and adolescents with achalasia. The success rate after a single dilatation was only $67 \%$ in a recent study. ${ }^{14}$ However the overall success rate after a maximum of 3 PBDs was $87 \%$ in the same study. Therefore, multiple sessions of dilatation may be required for optimal results of PBD. Moreover it may be technically difficult in younger patients $(<7$ years), and some patients may ultimately require surgery. Young patients do not respond well to PBD. ${ }^{14}$ The results of intra-esophageal botulinum toxin injection are not satisfactory in adults as well as pediatric achalasia patients. About one half of the patients suffer from recurrent symptoms after several months. ${ }^{15}$ Botulinum toxin should be used only for children with achalasia who are poor candidates for either pneumatic dilatation or surgery. Therefore, a safe and efficacious endoscopic modality of treatment is required in pediatric patients with achalasia.

LHM is the current surgical treatment of choice for children

Table 3. Objective Outcomes in Children Undergoing Per Oral Endoscopic Myotomy

\begin{tabular}{lccc} 
& & Post procedure & $P$-value \\
\hline Eckardt score $(\mathrm{n}=10)$, mean & $7.32 \pm 1.42$ & $1.74 \pm 0.67$ & 0.001 \\
Erosive esophagitis on EGD $(\mathrm{n}=10)$ & $0(0.0 \%)$ & $2(20.0 \%)$ & 0.001 \\
LES pressure on HRM $(\mathrm{n}=10), \mathrm{mmHg}$ & $36.64 \pm 11.08$ & $15.60 \pm 5.70$ & 0.001 \\
Percentage improvement in barium emptying at 5 min & & $63.70 \pm 4.46$ & \\
\hline
\end{tabular}

EGD, esophagogastroduodenoscopy; HRM, high-resolution manometry; LES, lower esophageal sphincter. 
and adolescents with achalasia. LHM appears to provide a more durable relief in symptoms than PBD at least in adult patients. ${ }^{16}$ In a recent case series, LHM was found to be safe and effective. However post-operative intervention (including balloon dilatation and redo myotomy) was required in $28 \%$ of patients on subsequent follow-up. ${ }^{17}$ Similarly, the recurrence of symptoms after LHM with fundoplication was $27 \%$ at a mean follow-up of 13 months in another series. ${ }^{18}$ Early and durable treatment is especially important in the pediatric population as treatment failure may lead to malnutrition and growth failure.

POEM is a novel technique for the treatment of achalasia. The procedure involves mucosal incision and entry into the submucosa, creation of a submucosal tunnel, actual myotomy, and finally closure of the mucosal incision. The term POEM was coined by Inoue et al, who initially reported its usefulness in 17 adult patients with achalasia. ${ }^{19}$ Since then, many studies have been published that demonstrate excellent short term outcomes and safety of POEM for achalasia. Reported complications include subcutaneous emphysema, mucosal injury, pneumothorax, pneumomediastinum, pleural effusion, and pneumoperitoneum. ${ }^{20}$ The incidence of these gas related adverse event is significantly less with the use of $\mathrm{CO}_{2}$ as it diffuses much faster as compared to air, and usually subside with conservative management. In our study, we observed gas related complications (including self limiting subcutaneous emphysema, retroperitoneal air requiring temporary stoppage of procedure) in 3 of our patients. All resolved with conservative management without any untoward consequences.

The data of POEM in children is very limited. The first case of POEM in children was recently reported in a 3-year-old girl with achalasia and Down's syndrome. The child had good symptom response when followed till 1 year. ${ }^{21}$ Another small series consisting of 3 children documented satisfactory outcome of POEM in pediatric achalasia. ${ }^{22}$ Since these initial reports, few more studies have been published demonstrating the safety and efficacy of POEM for treatment of achalasia in children and adolescents. ${ }^{23-25}$

We found POEM to be safe and effective in the pediatric age group, with no major adverse events. Complete resolution of symptoms along with significant fall in baseline LES pressure was noted in all the children after POEM (median follow-up 14 months). Previous procedures like LHM or PBD did not preclude the success to POEM. The response to POEM was durable in our study as evident by persistent symptom relief (Eckardt $\leq 3$ ) and objective parameters like timed barium esophagogram. None of the children required a second procedure like PBD and LHM.

The proposed advantages of POEM over LHM include shorter operative time, reduced intraprocedural blood loss, shorter hospital stay, and ability to extend myotomy depending on the type of achalasia. ${ }^{26-28}$

Moreover, as POEM does not involve the dissection of the diaphragmatic hiatus and division of the crura, the chances of post procedure gastroesophageal reflux disease are theoretically less than LHM. ${ }^{29}$ We observed gastroesophageal reflux symptoms in 3 of our patients, and two of them were found to have mild esophagitis on endoscopy. All were managed successfully with proton pump inhibitors. We acknowledge the fact that follow-up with $\mathrm{pH}$ studies are required to know the exact incidence of gastroesophageal reflux disease after POEM.

At present, the role of POEM is not established in pediatric patients with achalasia. POEM is minimally invasive with faster recovery as compared to LHM.

Excellent short term results warrant further evaluation of this novel technique in larger trials.

\section{Financial support: None.}

\section{Conflicts of interest: None.}

Author contributions: Zaheer Nabi, Mohan Ramchandani, D Nageshwar reddy, Santosh Darisetty, and Rama Kotla were involved in performing the POEM procedure and conducting the study; and Rakesh Kalapala and Radhika Chavan were involved in collection and interpretation of data and drafting the manuscript.

\section{References}

1. Goldblum JR, Whyte RI, Orringer MB, Appelman HD. Achalasia: a morphologic study of 42 resected specimens. Am J Surg Pathol 1994;18:327-337.

2. Franklin AL, Petrosyan M, Kane TD. Childhood achalasia: a comprehensive review of disease, diagnosis and therapeutic management. World J Gastrointest Endosc 2014;6:105-111.

3. Marlais M, Fishman JR, Fell JM, Haddad MJ, Rawat DJ. UK incidence of achalasia: an 11-year national epidemiological study. Arch Dis Child 2011;96:192-194.

4. Mayberry JF, Mayell MJ. Epidemiological study of achalasia in children. Gut 1988;29:90-93.

5. Hallal C, Kieling CO, Nunes DL, et al. Diagnosis, misdiagnosis, and associated diseases of achalasia in children and adolescents: a twelve-year single center experience. Pediatr Surg Int 2012;28:1211-1217.

6. Von Renteln D, Inoue H, Minami H, et al. Peroral endoscopic myotomy for the treatment of achalasia: a prospective single center study. Am J Gastroenterol 2012;107:411-417. 
7. Vaezi MF, Baker ME, Achkar E, Richter JE. Timed barium oesophagram: better predictor of long term success after pneumatic dilation in achalasia than symptom assessment. Gut 2002;50:765-770.

8. Eckardt AJ, Eckardt VF. Treatment and surveillance strategies in achalasia: an update. Nat Rev Gastroenterol Hepatol 2011;8:311-319.

9. Pandolfino JE, Kwiatek MA, Nealis T, Bulsiewicz W, Post J, Kahrilas PJ. Achalasia: a new clinically relevant classification by high-resolution manometry. Gastroenterology 2008;135:1526-1533.

10. Friedel D, Modayil R, Iqbal S, Grendell JH, Stavropoulos SN. Per-oral endoscopic myotomy for achalasia: an American perspective. World J Gastrointest Endosc 2013;5:420-427.

11. Ramchandani M, Nageshwar Reddy D. Peroral endoscopic myotomy: technique of mucosal incision. Clin Gastroenterol Hepatol 2014;12:900901.

12. Sharp NE, St Peter SD. Treatment of idiopathic achalasia in the pediatric population: a systematic review. Eur J Pediatr Surg 2016;26:143-149.

13. Hamza AF, Awad HA, Hussein O. Cardiac achalasia in children. Dilatation or surgery? Eur J Pediatr Surg 1999;9:299-302.

14. Di Nardo G, Rossi P, Oliva S, et al. Pneumatic balloon dilation in pediatric achalasia: efficacy and factors predicting outcome at a single tertiary pediatric gastroenterology center. Gastrointest Endosc 2012;76:927-932.

15. Hurwitz M, Bahar RJ, Ament ME, et al. Evaluation of the use of botulinum toxin in children with achalasia. J Pediatr Gastroenterol Nutr 2000;30:509-514.

16. Lopushinsky SR, Urbach DR. Pneumatic dilatation and surgical myotomy for achalasia. JAMA 2006;296:2227-2233.

17. Pachl MJ, Rex D, Decoppi P, et al. Paediatric laparoscopic Heller's cardiomyotomy: a single centre series. J Pediatr Surg 2014;49:289-292; discussion 292.

18. Askegard-Giesmann JR, Grams JM, Hanna AM, Iqbal CW, Teh S, Moir CR. Minimally invasive Heller's myotomy in children: safe and effective. J Pediatr Surg 2009;44:909-911.
19. Inoue $\mathrm{H}$, Minami H, Kobayashi $\mathrm{Y}$, et al. Peroral endoscopic myotomy (POEM) for esophageal achalasia. Endoscopy 2010;42:265-271.

20. Stavropoulos SN, Modayil RJ, Friedel D, Savides T. The International Per Oral Endoscopic Myotomy Survey (IPOEMS): a napshot of the global POEM experience. Surg Endosc 2013;27:3322-3338.

21. Maselli R, Inoue H, Misawa M, et al. Peroral endoscopic myotomy (POEM) in a 3-year-old girl with severe growth retardation, achalasia, and Down syndrome. Endoscopy 2012;44(suppl 2):E285-E287.

22. Familiari P, Marchese M, Gigante G, et al. Peroral endoscopic myotomy for the treatment of achalasia in children. J Pediatr Gastroenterol Nutr 2013;57:794-797.

23. Li C, Tan Y, Wang X, Liu D. Peroral endoscopic myotomy for treatment of achalasia in children and adolescents. J Pediatr Surg 2015;50:201-205.

24. Tang X, Gong W, Deng Z, et al. Usefulness of peroral endoscopic myotomy for treating achalasia in children: experience from a single center. Pediatr Surg Int 2015;31:633-638.

25. Chen WF, Li QL, Zhou PH, et al. Long-term outcomes of peroral endoscopic myotomy for achalasia in pediatric patients: a prospective, singlecenter study. Gastrointest Endosc 2015;81:91-100.

26. Bhayani NH, Kurian AA, Dunst CM, Sharata AM, Rieder E, Swanstrom LL. A comparative study on comprehensive, objective outcomes of laparoscopic Heller myotomy with per-oral endoscopic myotomy (POEM) for achalasia. Ann Surg 2014;259:1098-1103.

27. Hungness ES, Teitelbaum EN, Santos BF, et al. Comparison of perioperative outcomes between peroral esophageal myotomy (POEM) and laparoscopic Heller myotomy. J Gastrointest Surg 2013;17:228-235.

28. Kumbhari V, Tieu AH, Onimaru M, et al. Peroral endoscopic myotomy (POEM) vs laparoscopic Heller myotomy (LHM) for the treatment of Type III achalasia in 75 patients: a multicenter comparative study. Endosc Int Open 2015;3:E195-E201.

29. Dunst CM, Kurian AA, Swanstrom LL. Endoscopic myotomy for achalasia. Adv Surg 2014;48:27-41. 